\title{
Tumores das Células da Granulosa dos Ovários: Estudo de 24 Casos
}

\author{
Granulosa Cell Tumors of the Ovary: A Study of 24 Cases \\ Artur Lício Rocha Bezerra, Felipe Rinalo Barbosa Lorenzato
}

\begin{abstract}
RESUM0
Objetivos: avaliar estadiamento, procedimentos cirúrgicos e evolução dos tumores das células da granulosa (TCG) dos ovários, dando ênfase à possibilidade de cirurgia conservadora (ooforectomia unilateral).

Métodos: este é um estudo de coorte retrospectivo no qual foram incluídas 24 pacientes com TCG tratadas no período de janeiro de 1994 a janeiro de 2004. Foram analisadas variáveis de interesse, tais como idade da paciente, sintomatologia, tamanho tumoral ao exame fisico, estadiamento, modalidades de tratamento (tipos de cirurgia e adjuvância com quimioterapia e/ou radioterapia) e prognóstico. As associações entre as variáveis foram avaliadas pelo teste do chi-quadrado e para significância estatística considerou-se $p<0,05$, sempre se descrevendo o teste exato bicaudado de Fisher.

Resultados: a idade das 24 pacientes variou de 30 a 82 (média 51,7) anos. O sintoma mais freqüente foi a dor pélvica $(n=10 ; 41,7 \%)$. Quatorze pacientes $(58,3 \%)$ apresentavam estádio clínico (EC) Ic, 5 (20,8\%) EC IIIc e $5(20,8 \%)$ EC Ia. Estadiamentos mais avançados foram significativamente associados com o achado de massas pélvicas palpáveis $(p<0,04)$. Hiperplasias endometriais típicas foram encontradas em 3 (25\%) de 12 espécimes de histerectomia. O tempo de seguimento variou de 2 a 114 (média 42,5) meses. Dentre as 16 (66,7\%) pacientes vivas e sem doença, 4 eram EC Ia (25\%), 11 EC Ib $(68,8 \%)$ e 1 EC Ic (6,3\%), enquanto todas as pacientes que faleceram eram EC IIIc ( $p=0,0008)$. Todas as 6 pacientes (25\%) que se submeteram à cirurgia conservadora estavam vivas e sem recidiva.

Conclusão: embora os TCG geralmente apresentem baixa agressividade, o estadiamento continua sendo o principal fator prognóstico e assim ele determina a opção de cirurgia conservadora e do uso de tratamento adjuvante.
\end{abstract}

PALAVRAS-CHAVE: Ovário. Câncer. Tumores de células da granulosa.

\section{Introdução}

Os tumores das células da granulosa (TCG) são raros, representando 2 a 3\% de todas as malignidades ovarianas. Compreendem, no entanto, $70 \%$ dos tumores do estroma e cordões sexuais, sendo o tipo mais comum nesta categoria ${ }^{1}$. São compostos de células da granulosa isoladamente ou em combinação com outros elementos do

Hospital do Câncer de Pernambuco

Correspondência:

Rua Teles Júnior, 155, apt. 301 - Rosarinho

52050-040 - Recife - PE

Fone: (81) 3426-7670 - 9996-8378

e- mail: artur.licio@terra.com.br estroma, mais freqüentemente células da teca e raramente células de Sertoli ou Leydig ${ }^{2}$.

Foram descritos dois subtipos de TCG: as formas juvenil (TCGJ) e adulta (TCGA). Cerca de 95\% dos casos são TCGA, acometendo mulheres na peri ou pós-menopausa, com média de idade de 50 anos. Os sintomas, em geral, decorrem da presença de massa tumoral ou de produção hormonal (17- $\beta$ estradiol) pela neoplasia ${ }^{1}$.

Os TCG são considerados pouco agressivos. Em contraste com as neoplasias epiteliais, apresentam bom prognóstico, refletindo uma propensão desses tumores de permanecerem localizados, com taxas de sobrevida no estádio I superiores a $95 \%{ }^{1}$. Existem relatos, no entanto, de invasão de vasos 
sanguíneos e linfáticos, com metástases para fígado, pulmão e rim, além da presença de implantes peritoneais ${ }^{3,4}$. As recorrências em geral são tardias, com tempo médio de aparecimento de 5 a 10 anos após o diagnóstico, e ocorrem na maioria das vezes no próprio abdome ${ }^{1}$.

Os principais fatores prognósticos relatados são o estadiamento da lesão, a presença ou ausência de rotura da cápsula tumoral e o grau de diferenciação da neoplasia. Outros fatores prognósticos anatomopatológicos citados são o índice mitótico e a presença de embolização vascular por células neoplásicas ${ }^{1,4}$.

Os TCG são tratados fundamentalmente com cirurgia, que pode ser seguida de tratamento adjuvante nos casos de doença extra-ovariana. Não existe consenso, no entanto, para as indicações de radio ou quimioterapia ${ }^{5}$. Considerando a raridade da neoplasia e a falta de estudos sobre o assunto na literatura nacional, o objetivo do presente estudo foi analisar o estadiamento, evolução clínica e tratamento de 24 casos de TCG, com ênfase na possibilidade de cirurgia conservadora.

\section{Pacientes e Métodos}

Foi realizado estudo de 24 pacientes portadoras de TCG tratadas no Departamento de Cirurgia Pélvica do Hospital do Câncer de Pernambuco no período de janeiro de 1994 a janeiro de 2004 . Destas, apenas duas submeteram-se ao tratamento cirúrgico primário em outros hospitais, sendo encaminhadas com relatório médico para tratamento adjuvante e seguimento.

As 22 pacientes operadas na instituição seguiram a rotina pré-operatória, com exames hematológicos, bioquímicos, radiológicos (Rx tórax) e ultra-sonográficos. A presença de massa tumoral ovariana mista foi o aspecto ultra-sonográfico mais comum. Em nenhum caso, no entanto, o exame sugeriu o diagnóstico de TCG. Não se observou também nenhum caso de espessamento endometrial.

Todas as pacientes foram estadiadas após análise do achado cirúrgico, seguindo os critérios atuais da Federação Internacional de Ginecologia e Obstetrícia (FIGO) ${ }^{6}$. Em virtude das dificuldades técnicas para o diagnóstico histopatológico intraoperatório (congelação) nessa neoplasia, a definição dos casos foi realizada por meio do exame convencional de parafina.

As lâminas foram revistas no Departamento de Patologia do referido hospital, sendo o diagnóstico de TCG baseado na proliferação de células relativamente monomórficas, de núcleos ovóides, regulares, exibindo, em alguns setores, sulcos longitudinais; essas células se agrupavam formando massas irregulares com presença de arranjos macro ou microfoliculares e ocasionais corpúsculos de Call-Exner. Nos casos em que foi realizada histerectomia total, investigou-se a associação de hiperplasia endometrial.

Foram estudadas características clínicas, como idade, queixas, tipo de cirurgia, tratamento adjuvante e evolução.

O acompanhamento das pacientes foi realizado em consultas ambulatoriais, trimestrais do primeiro ao terceiro ano após a cirurgia, semestrais no quarto ano e anuais a partir do quinto ano.

Para arquivo e registro das informações coletadas foi criado formulário específico. A mensuração das variáveis foi expressa pelas médias, ao passo que a correlação entre algumas variáveis foi avaliada pelo teste do $\chi^{2}$ em tabelas de contingência de dupla entrada. Quando em tabela 2 × 2 uma célula teve o número inferior a 5 , foi utilizado o teste exato de Fisher bi-caudado para descrição do valor de p. Os $\chi^{2}$ descritos foram sempre o Yates corrigido e nos intervalos de confiança utilizamos os limites exatos. Foi aceito $\mathrm{p}<0,05(95 \%)$ como critério para rejeição da hipótese de nulidade.

O presente estudo foi revisado e aprovado pela Comissão de Ética do Hospital do Câncer de Pernambuco antes do início da coleta de dados.

\section{Resultados}

A idade das 24 pacientes variou de 30 a 82 anos (média 51,7). Quando o TCG foi diagnosticado, 11 pacientes $(45,8 \%)$ tinham menos de 50 anos e encontravam-se na pré-menopausa e 13 $(54,2 \%)$ tinham mais de 50 anos e eram menopausadas (Tabela 1). A dimensão dos tumores ovarianos foi variável (menor que $2 \mathrm{~cm}$ até $40 \mathrm{~cm}$; média: $13 \mathrm{~cm}$ ).

O estadiamento mais freqüente foi Ic, encontrado em 14 pacientes (58,3\%). A inclusão nesse estadiamento foi decorrente da presença de tumor na superfície do ovário. Cinco $(20,8 \%)$ tinham estadio IIIc, com disseminação e implantes macroscópicos na cavidade abdominal e em 5 $(20,8 \%)$ casos a lesão restringia-se ao interior do parênquima ovariano (estadio Ia) (Tabela 2). A citologia do líquido peritoneal foi positiva nos 5 casos de estadiamento IIIc e negativa em todos os casos de estadiamento I. 
Tabela 1 - Características clínicas das 24 pacientes portadoras de tumores das células da granulosa dos ovários.

\begin{tabular}{lcc}
\hline Características clínicas & $\mathbf{N}^{0}$ de pacientes & $\%$ \\
\hline Idade & 11 & \\
$\quad$ Até 50 anos & 13 & 54,8 \\
$\quad$ Mais de 50 anos & & \\
Sintomas ao diagnóstico & 10 & 41,7 \\
$\quad$ Dor pélvica & 4 & 16,7 \\
Aumento do volume abdominal & 6 & 25 \\
Sangramento vaginal & 2 & 8,3 \\
Tumor pélvico & 1 & 4,2 \\
Assintomática & 1 & 4,2 \\
Sem informação & & 8 \\
Exame físico inicial & 2 & 92 \\
Normal & 22 & \\
Tumor pélvico & & \\
\hline
\end{tabular}

As cirurgias realizadas foram histerectomia total com anexectomia bilateral $(n=8 ; 33,3 \%)$, citorredução $(\mathrm{n}=6 ; 25 \%)$, ooforectomia unilateral $(\mathrm{n}=6 ; 25 \%)$, histerectomia total com anexectomia unilateral $(\mathrm{n}=3 ; 12,5 \%)$ e laparotomia com biópsia $(\mathrm{n}=1 ; 4.2 \%)$. O útero foi analisado em 12 casos (incluindo-se uma paciente submetida à citorredução na qual foi feita histerectomia) (Tabela 2). Foram detectados 3 pólipos endometriais $(25 \%), 6$ leiomiomas $(50 \%)$ e 3 hiperplasias endometriais típicas $(25 \%)$.

Tabela 2 - Estadiamento (FIGO) e procedimentos cirúrgicos realizados nas 24 pacientes portadoras de tumores das células da granulosa dos ovários.

\begin{tabular}{lcc}
\hline Variável & Pacientes & $\%$ \\
\hline Estadiamento & 5 & \\
Ia & 14 & 20,8 \\
IC & 5 & 58,3 \\
IIIC & & 20,8 \\
Procedimentos cirúrgicos & 8 & 33,3 \\
Hta + sob & 6 & 25 \\
Citorredução & 6 & 25 \\
Ooforectomia unilateral & 3 & 12,5 \\
$\quad$ Hta + sou & 1 & 4,2 \\
Laparotomia com biópsia &
\end{tabular}

$\mathrm{Hta}+\mathrm{sob}$ : histerectomia total com salpingooforectomia bilateral; $\mathrm{Hta}+\mathrm{sou}$ : histerectomia total com salpingooforectomia unilateral.

Os estadiamentos mais avançados foram significativamente associados com o achado, ao exame físico inicial, de massas pélvicas palpáveis
( $\mathrm{p}<0,04)$. O estádio clínico Ia foi encontrado nas duas pacientes cujo exame clínico foi normal e em 3/22 $(13,6 \%)$ daquelas com tumor pélvico (Tabela 3).

Tabela 3 - Associação entre o exame físico inicial e 0 estadiamento dos tumores das células da granulosa.

\begin{tabular}{lccr}
\hline Estadiamento & \multicolumn{2}{c}{ Exame ginecológico } & Total \\
& Tumor palpável & Exame normal & \\
\hline$>$ la & 19 & 0 & 19 \\
la & 3 & 2 & 5 \\
Total & 22 & 2 & 24 \\
\hline
\end{tabular}

$\mathrm{RR}=1,67[0,81 ; 3,41] \cdot \chi^{2}=3,88 ; p<0,04$.

Quimioterapia adjuvante foi aplicada a sete pacientes $(29,2 \%$ ) (5 com estádio Ic e 2 com estádio IIIc), sem haver uma padronização no tratamento. O esquema mais utilizado foi a associação de bleomicina (20 unidades $/ \mathrm{m}^{2}$ nos dias 1,8 e 15), etoposide ( $100 \mathrm{mg} / \mathrm{m}^{2}$ do dia 1 ao dia 5 ) e cisplatina (20 mg/ $\mathrm{m}^{2}$ do dia 1 ao dia 5), repetido a cada três semanas num total de 3 ciclos.

O tempo de seguimento variou de 2 a 114 meses (média: 42,5). Houve quatro óbitos pela doença: uma paciente faleceu no pós-operatório imediato (choque hipovolêmico) após realização de ooforectomia, enterectomia e cistectomia parcial por infiltração tumoral no intestino e bexiga. Duas pacientes faleceram dois meses após a cirurgia. Nesses dois casos o tumor foi considerado irressecável, havendo comprometimento múltiplo de órgãos abdominais. A outra paciente que evoluiu para óbito havia sido submetida à cirurgia citorredutora, considerada ótima, e quimioterapia adjuvante com BEP, tendo apresentado recidiva na cúpula vaginal com 17 meses de seguimento. Foi tratada com radioterapia, mas faleceu três meses após o diagnóstico da recidiva.

Como demonstra a Tabela 4 , houve associação significativa entre o estadiamento e a sobrevida das pacientes. Considerando as dezesseis pacientes $(66,7 \%)$ que estavam assintomáticas ao final do seguimento de em média 42,5 meses, 4 eram estádio clínico Ia $(25 \%), 11 \mathrm{Ib}(68,8 \%)$ e $1(6,3 \%)$ Ic, ao passo que todas as pacientes que evoluíram para óbito pela neoplasia apresentavam estádio clínico IIIc $(\mathrm{p}=0,0008)$. Houve um óbito por outra causa (infarto do miocárdio) e três pacientes foram perdidas no seguimento. As seis pacientes (25\%) que se submeteram a cirurgias conservadoras (ooforectomia unilateral) estavam vivas e sem recidiva. 
Tabela 4 - Associação entre 0 estadiamento e a sobrevida das 24 pacientes com tumores das células da granulosa dos ovários.

\begin{tabular}{lccr}
\hline Estadiamento & \multicolumn{2}{c}{ Situação clínica } & Total \\
& Óbito & Viva sem doença & \\
\hline EC $>$ IC & 4 & 1 & 5 \\
EC $\leq$ IC & 0 & 16 & 16 \\
Total & 4 & 17 & 21 \\
\hline
\end{tabular}

Dois casos com estádio Ic e 1 caso com estádio la perderam-se do seguimento. $\chi^{2}=11,05$ $p=0,0008$.

\section{Discussão}

Em virtude de se tratar de neoplasia rara, com incidência de 1/1.000.000 mulheres nos Estados Unidos $^{5}$, os estudos sobre TCG apresentam pequeno número de casos com avaliação estatística e as revisões em geral incluem pacientes de vários centros médicos. É comum também se encontrarem na literatura estudos com casos isolados ${ }^{3,7}$. O número de pacientes estudados na presente série (24) é representativo, principalmente se considerarmos serem oriundos de uma única instituição, estando na média dos demais estudos analisados ${ }^{2,4,8,9}$.

$\mathrm{O}$ pico de incidência dos TCG ocorre entre 45 e 55 anos. A idade média ao diagnóstico em uma análise conjunta de 750 casos foi de 51 anos $^{5}$, dados semelhantes ao presente estudo (idade média: 51,7 anos). Os TCG que ocorrem em crianças e adultos jovens apresentam características clínicas distintas, como presença de pseudopuberdade precoce, elevados níveis séricos de estradiol e progesterona e aumento do volume abdominal como queixa inicial ${ }^{10}$. Nos estadiamentos mais avançados são caracteristicamente agressivos, com curtos períodos para recorrência e morte. $\mathrm{O}$ tempo médio para recorrência dos TCG na forma adulta, ao contrário, é longo, variando de 4 a 6 anos. Existem relatos de recorrência 19 e 37 anos após o tratamento primário ${ }^{3,7}$.

A maioria das pacientes portadoras de TCG apresentam sintomas inespecíficos, como dor e distensão abdominal, fato também observado neste estudo. Em virtude de secreção hormonal pelo tumor, podem haver manifestações endócrinas como menometrorragias, sangramentos intermenstruais e sangramentos após a menopausa ${ }^{1}$. Segundo Hartmann et al. $^{5}$, metade das pacientes desenvolve hiperplasia endometrial atípica e cerca de $25 \%$ evoluem com adenocarcinomas do endométrio. No presente estudo foi detectada hiperplasia endometrial típica em 3/12 (25\%) casos. Esses números estão com certeza subavaliados porque não foi realizada histerectomia nas demais pacientes.
Apesar dos sinais clínicos de hiperestrogenismo em muitas pacientes, não existe sistematização na investigação pré-operatória, com dosagem de estrógenos, porque os níveis desse hormônio são usualmente muito baixos e não servem para serem usados no seguimento ${ }^{5}$. Outro marcador, a inibina, peptídeo hormonal que é estimulado pelo FSH durante a fase folicular e LH na fase luteal, tem sido avaliado em alguns estudos, mas sua sensibilidade e especificidade ainda não estão estabelecidas ${ }^{11,12}$. Segundo Hildebrandt et al. ${ }^{12}$, os niveis de inibina podem estar aumentados em 17 a 80\% dos cânceres ovarianos. Em virtude da raridade dessa neoplasia, a utilização de marcadores tumorais em programas de screening é inviável A utilização dessas substâncias é mais útil na monitorização para eventuais recorrências.

Embora o estadiamento dos TCG e dos tumores epiteliais do ovário seja o mesmo (FIGO), a evolução e o crescimento dessas duas neoplasias são diferentes. Enquanto os tumores epiteliais originam implantes peritoneais independentemente do tamanho do tumor primário, sendo diagnosticados geralmente em estádios IIIc, os TCG crescem como massas pélvicas, de tamanhos variados, na sua maioria detectados no estádio $\mathrm{I}^{13}$. Podem, no entanto, apresentar disseminação semelhante aos tumores epiteliais, com metástases intra-abdominais ou a distância. No estudo de Fujimoto et al. ${ }^{4}$, por exemplo, $7 / 27$ (25,9\%) pacientes tiveram recorrência da doença, sendo 5 com doença extrapélvica, especialmente no fígado.

No presente estudo os tumores tiveram, em média, $13 \mathrm{~cm}$, semelhante ao relato de Malmstrom et al. ${ }^{14}$, no qual a maioria das pacientes apresentaram tumor entre 8 e $14 \mathrm{~cm}$. Os TCG podem variar bastante de tamanho, podendo se apresentar como pequenas massas até grandes neoplasias que preenchem toda a pélvis ${ }^{1}$.

Cerca de $80 \%$ dos tumores da presente série foram diagnosticados no estádio I, semelhante ao observado em outras séries ${ }^{2,14}$. Vinte e duas pacientes $(91,7 \%)$ deste estudo já apresentavam um exame físico positivo ao diagnóstico, com o achado de massas pélvicas palpáveis, evidenciando atraso no diagnóstico. A ultra-sonografia com dopplervelocimetria, no entanto, não parece ajudar na diferenciação pré-operatória entre os TCG e os tumores epiteliais; a presença de septações, papilas ou cistos complexos, além dos índices do fluxo vascular, são semelhantes entre os dois tipos de tumor ${ }^{15}$.

O estadiamento continua sendo o principal fator prognóstico nos TCG ovarianos. Tumores restritos ao ovário (ECI) estão associados com 
melhor sobrevida e menor índice de recorrências $^{16}$. Lauszus et al. ${ }^{2}$ relatam sobrevida atuarial a 5 e 10 anos de 94 e $82 \%$, respectivamente, em 37 pacientes portadoras de TCG ECI. Dois outros grandes estudos na literatura não relataram diferença na sobrevida quando a abordagem inicial foi conservadora ou quando foi realizada histerectomia total com salpingo-ooforectomia bilateral nos estadiamento iniciais ${ }^{17,18}$. Em mulheres jovens e com desejo de engravidar, portanto, a salpingo-ooforectomia unilateral tem sido indicada nos TCG estádio I.

As seis pacientes do presente estudo que apresentavam tumor restrito aos ovários foram submetidas a cirurgias conservadoras e estavam vivas sem doença ao final do seguimento (média de 42,5 meses), ao passo que todas as pacientes que evoluíram para óbito apresentavam doença disseminada no peritônio (estádio III). Consideramos, no entanto, que o tempo de seguimento deste estudo é pequeno, o que dificulta uma melhor análise das taxas de recidiva. As recorrências dos TCG podem ocorrer muito tempo após o tratamento do tumor primário ${ }^{7}$.

Nos tumores em estadiamento avançado (III e IV) a cirurgia citorredutora está indicada, de forma semelhante à abordagem empregada para as neoplasias epiteliais, com impacto negativo na sobrevida quando existe tumor residual macroscópico pós-operatório ${ }^{8}$. Nesses casos em geral utiliza-se tratamento adjuvante, embora não haja consenso quanto à forma de abordagem.

A radioterapia é sugerida em alguns estudos, mas parece não ter nenhum benefício nos estadiamentos avançados ou nas recidivas ${ }^{5,9}$, ao passo que não existem estudos prospectivos que determinem a eficácia da quimioterapia em virtude da raridade da neoplasia e do seu curso clínico prolongado. A utilização de bleomicina, etoposida e cisplatina vem sendo avaliada como terapia de primeira linha para estas pacientes ${ }^{5}$. Al-Badawi et al. ${ }^{8}$, analisando 55 pacientes com estadiamento III e IV, concluíram que o uso de quimioterapia adjuvante não melhorou a sobrevida daquelas pacientes que apresentavam doença residual após a cirurgia citorredutora. Colombo et al. ${ }^{19}$, por outro lado, relatam sucesso com cisplatina, vimblastina e bleomicina em 11 pacientes, cinco das quais com nenhuma ou mínima doença residual após cirurgia citorredutora para estádio clínico III e IV. Não foi possivel avaliar a resposta à quimioterapia nas 7 pacientes do presente estudo que se submeteram a este tratamento, em virtude da falta de padronização na indicação clínica.

Concluindo, nossos achados enfatizam a importância do toque vaginal combinado, na opor- tunidade do exame ginecológico, devido à associação significativa entre a presença de tumoração anexial palpável e TCG com estádio maior que 1a. Embora os TCG sejam considerados como de baixa agressividade, o estadiamento da neoplasia continua sendo o principal fator prognóstico e oferece a opção de cirurgias conservadoras.

\section{ABSTRACT}

Objective: to evaluate staging, surgical procedures and outcome of ovarian granulosa cell tumors (GCT), with emphasis in the possibility of conservative surgery (unilateral ooforectomy).

Methods: this is a retrospective cohort study in which 24 patients treated with GCT during the period of January 1994 to January 2004 were included. Variables of interest such as patient's age, symptoms, tumor size on physical examination, staging, treatment modalities (types of surgery and of adjuvant chemotherapy and/or radiotherapy) and prognosis have been analyzed. The chi-square test was used to check the association between variables, and the level of significance was set at $p<$ 0.05 , always describing the two-tailed Fisher exact test.

Results: the patients' age varied from 30 to 82 (mean 51.7) years old. The most frequently referred symptom was pelvic pain $(n=10 ; 41.7 \%)$. Fourteen patients $(58.3 \%)$ presented clinical stage (CS) Ic, 5 (20.8\%) CS IIIc and 5 (20.8\%) CS Ia. More advanced clinical stages were significantly associated with palpable pelvic masses ( $p<0.04)$. Endometrial hyperplasias were found in 3 (25\%) of the 12 hysterectomy specimens. The follow-up period varied from 2 to 114 (mean 42.5) months. Among the $16(66.7 \%)$ surviving and disease-free patients, 4 had CS Ia (25\%), 11 CS Ib $(68.8 \%)$ and 1 CS Ic (6.3\%), whereas all patients who died had CS IIIc $(p=0.0008)$. All 6 patients $(25 \%)$ that underwent conservative surgery were alive and without recurrence. Conclusion: although GCT usually present low aggressiveness, the clinical staging continues to be the main prognostic factor and hence it determines the option for conservative surgery and the use of adjuvant therapy.

KEYWORDS: Ovary. Cancer. Granulosa cell tumors.

\section{Referências}

1. Segal R, DePetrillo AD, Thomas G. Clinical review of adult granulosa cell tumors of the ovary. Review. Gynecol Oncol 1995; 56:338-44.

2. Lauszus FF, Petersen AC, Greisen J, Jakobsen A. Granulosa cell tumor of the ovary: a populationbased study of 37 women with stage I disease. Gynecol Oncol 2001; 81:456-60. 
3. Dubuc-Lissoir J, Berthiaume MJ, Boubez G, Van Nguyen T, Allaire G. Bone metastasis from a granulosa cell tumor of the ovary. Gynecol Oncol 2001; 83:400-4.

4. Fujimoto T, Sakuragi N, Okuyama K, et al. Histopathological prognostic factors of adult granulosa cell tumors of the ovary. Acta Obstet Gynecol Scand 2001; 80:1069-74.

5. Hartmann LC, Young RH, Evans MP, Podratz KC. Ovarian sex cord-stromal tumors. In: Hoskins WJ, Perez CA, Young RC, editors. Principles and Practice of Gynecologic Oncology. $2^{\text {nd }}$ ed. Philadelphia: Lippincott Raven; 1997. p. 1003-24.

6. Benedet JL, Bender H, Jones H $3^{\text {rd }}$, Ngan HY, Pecorelli S. FIGO staging classification and clinical practice guidelines in the management of gynecological cancers. Int J Gynaecol Obstet 2000; 70:209-62.

7. Hines JF, Khalifa MA, Moore JL, Fine KP, Lage JM, Barnes WA. Recurrent granulosa cell tumor of the ovary 37 years after initial diagnosis: a case report and review of the literature. Gynecol Oncol 1996; 60:484-8.

8. Al-Badawi IA, Brasher PMA, Ghatage P, Nation JG, Schepansky A. Postoperative chemotherapy in advanced ovarian granulosa cell tumors. Int J Gynecol Cancer 2002; 12:119-23.

9. Wolf JK, Mullen J, Eifel PJ, Burke TW, Levenback C, Gershenson DM. Radiation treatment of advanced or recurrent granulosa cell tumor of the ovary. Gynecol Oncol 1999; 73:35-41.

10.Lack EE, Perez-Atayde AR, Murthy AS, Goldstein DP, Crigler JF Jr, Vawter GF. Granulosa theca cell tumors in premenarchal girls: a clinical and pathologic study of ten cases. Cancer 1981; 48:1846-54.
11.Fuller PJ, Chu S, Fikret S, Burger HG. Molecular pathogenesis of granulosa cell tumours. Mol Cell Endocrinol 2002; 191:89-96.

12.Hildebrandt RH, Rouse RV, Longacre TA. Value of inhibin in the identification of granulosa cell tumors of the ovary. Hum Pathol 1997; 28:1387-95.

13.Fox H. Pathologic prognostic factors in early stage adult-type granulosa cell tumors of the ovary. Int $\mathrm{J}$ Gynecol Cancer 2003; 13:1-4.

14. Malmstrom H, Hogberg T, Risberg B, Simonsen E. Granulosa cell tumors of the ovary: prognostic factors and outcome. Gynecol Oncol 1994; 52:50-5.

15.Sharony R, Aviram R, Fishman A, et al. Granulosa cell tumors of the ovary: do they have any unique ultrasonographic and color Doppler flow features? Int J Gynecol Cancer 2001; 11:229-33.

16.Vesco KK, Carney ME. Granulosa cell tumor of the ovary: extensive late recurrence after initial occult microscopic disease. Obstet Gynecol 2002; 99:888-91.

17.Sjostedt S, Wahlen T. Prognosis of granulosa cell tumors. Acta Obstet Gynecol Scand 1961; 40 (Suppl 6):1-26.

18.Bjorkholm E, Silfversward C. Prognostic factors in granulosa cell tumors. Gynecol Oncol 1981; 11:261-74.

19.Colombo N, Sessa C, Landoni F, Sartori E, Pecorelli $\mathrm{S}$, Mangioni C. Cisplatin, vinblastine, and bleomycin combination chemotherapy in metastatic granulosa-cell tumor of the ovary. Obstet Gynecol 1986; 67:265-8.

Recebido em: 8/7/2004 Aceito com modificações em: 2/9/2004 